\title{
Fabrication and characterization of plasmonic band-stop filter using Ag grating
}

\author{
Atsushi Motogaito ${ }^{1, *}$, Ryoga Tanaka $^{1}$, and Kazumasa Hiramatsu ${ }^{1}$ \\ ${ }^{1}$ Mie University, Graduate School of Engineering, 1577 Kurima-machiya-cho Tsu, Japan
}

\begin{abstract}
This study proposes a plasmonic band-stop filter with surface plasmon resonance in a doublelayer wire grid structure targeting short-wavelength visible and near-ultraviolet regions for applications in ultraviolet photography. Using $\mathrm{Ag}$ and $\mathrm{Al}$, the rigorous coupling wave of analysis method revealed that the maximum absorption was approximately $90 \%$ at $450 \mathrm{~nm}$ and $375 \mathrm{~nm}$. The experiments using Ag produced similar results in a simulation. These results demonstrate that plasmonic band-stop filters in the visible and near-UV region can be realized at $450 \mathrm{~nm}$ and $375 \mathrm{~nm}$ using $\mathrm{Ag}$ or $\mathrm{Al}$.
\end{abstract}

\section{Introduction}

Ultraviolet (UV) photography is used in various fields, including medicine, science, engineering, criminal investigation, and visual arts. The most popular method of photographing is to expose UV rays to an imaging film or device. The most convenient and simple UV light source is direct sunlight. However, the intensity of the UV rays is highly dependent on the atmospheric conditions. For example, in sunny and dry highlands, the UV intensity of sunlight is high. Electronic flashes are additional examples of UV light sources that can be used in everyday life. Surface reflection by an aluminum reflector can be used for more effective irradiation of UV rays by an electric flash.

In using non-monochromatic light for UV photography, it is necessary to prepare appropriate filters to transmit or stop transmitting a specific wavelength. To solve this problem, we propose a plasmonic filter using the propagating surface plasmon polariton in a wire grid (WG) structure. In a previous study, experiments demonstrated by designing the structure of the doublelayer WG structure, the extraordinary transmission can be achieved at a specific incident angle and light wavelength $[1,2]$. In addition, results of transmittance mapping indicate that transmission phenomena can be explained by the propagating surface plasmon polariton in the double-layer WG structure.

If the conditions of the structure for absorbing light can be determined, the WG structure can be used as a band-stop filter. In a previous study, $\mathrm{Hu}$ simulated extraordinary transmission by a nanohole array in the UV region [3]. In addition, Jaksic fabricated a band-pass filter for the UV region by surface-plasmon-polaritonassisted metal-dielectric multilayers [4]. Mazulquim fabricated a band-pass color filter enabled by resonant modes and plasmons near the Rayleigh anomaly [5]. Furthermore, Liang fabricated plasmonic structural color thin films through the aluminum oxide process in an anodic aluminum oxide thin film [6].

In this study, we propose band-stop filters that stop transmitting light at $450 \mathrm{~nm}$ and $375 \mathrm{~nm}$ using extraordinary absorption by propagating surface plasmon resonance of the Ag double-layer WG structure.

\section{Design and fabrication method}

As illustrated in Fig. 1, the double-layer WG structures were designed and fabricated on a glass substrate. The light wavelength was 450 and $375 \mathrm{~nm}$. The thickness of the $\mathrm{Ag}$ and $\mathrm{Al}$ layer and the electron beam (EB) resist are presented in Table 1. Reflectance mapping was obtained by the rigorous coupling wave of analysis method using MATLAB-based commercial software (rcda-1d [SourceForage, http://rcwa1d.sourceforge.net/]) by arranging the calculation results of the transmittance with respect to the incident angle obtained for each period. The double-layer WG structure was fabricated using EB lithography (EBL) and direct current (DC) magnetron sputtering. It should be noted that the transmittance, reflectance, and absorption of $\mathrm{p}$ polarized light depends on the incidence angle.

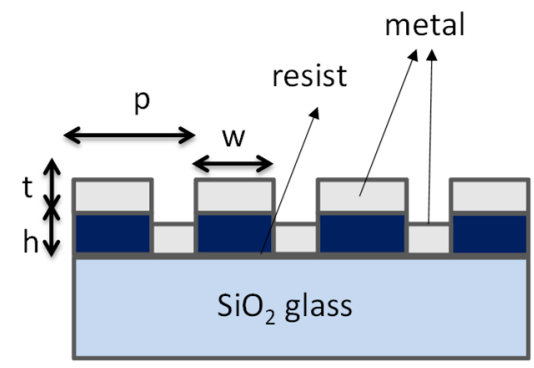

Fig. 1. Schematic drawing of double-layer wire grid structure.

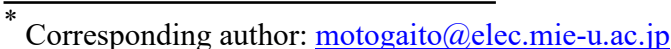


Table 1. Parameter of wire grid structures.

\begin{tabular}{|c|c|c|c|c|}
\hline $\begin{array}{c}\text { Wavelength } \\
\lambda(\mathrm{nm})\end{array}$ & \multicolumn{2}{|c|}{450} & \multicolumn{2}{c|}{375} \\
\hline Metal & $\mathrm{Ag}$ & $\mathrm{Al}$ & $\mathrm{Ag}$ & $\mathrm{Al}$ \\
\hline $\begin{array}{c}\text { Period } p \\
(\mathrm{~nm})\end{array}$ & 350 & 350 & 350 & 350 \\
\hline $\begin{array}{c}\text { Width of } \\
\text { resist } w \\
(\mathrm{~nm})\end{array}$ & 262.5 & 122. & 182.0 & 287.0 \\
\hline $\begin{array}{c}\text { duty ratio } \\
w / p\end{array}$ & 0.75 & 0.35 & 0.52 & 0.82 \\
\hline $\begin{array}{c}\text { Thickness } \\
\text { of resist } h \\
(\mathrm{~nm})\end{array}$ & 100 & 100 & 100 & 100 \\
\hline
\end{tabular}

\section{Results and discussion}

Fig. 2 presents the angle spectra of the absorption at 450 nm. The results demonstrate that the absorption peak occurred together with the reflectance dip at $16.4^{\circ}$ and that the maximum absorption was $91.2 \%$. In addition, in the experiment, a peak in the absorption rate was observed at the almost same angle. In the case of $\mathrm{Al}$, the absorption peak occurred at almost the same angle. The structure using Ag was sharper; however, the average absorption was larger in the structure using Al. These results demonstrate a structure capable of absorbing light with a wavelength of $450 \mathrm{~nm}$.

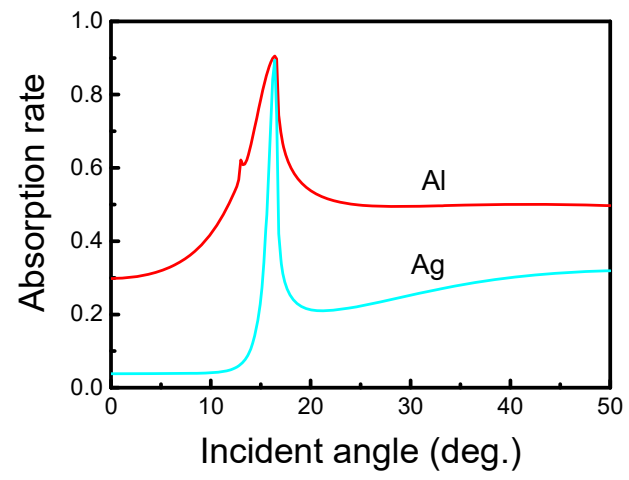

Fig. 2. Angle spectra of absorption rate at $450 \mathrm{~nm}$.

To consider the peak of the absorptance, Fig. 3 presents the reflectance color mapping, in which the abscissa axis represents the structural period and the ordinate axis represents the incident angle. Here the two-layer Ag WG structure is used as an example. This figure reveals the dispersion relation, and the curve formed by the low reflectance corresponds to the excitation curve of the surface plasmon. The incident angle of $16.4^{\circ}$ at which the absorption peak (reflectance dip) occurred in the structure using $\mathrm{Ag}$ (structure period $350 \mathrm{~nm}$ ) is on the excitation curve. Therefore, the generated abnormal absorption phenomenon can be considered an effect of surface plasmons. This phenomenon was also confirmed in the structure using Al. The absorption characteristics at $375 \mathrm{~nm}$ were also simulated. At $4.0^{\circ}$, a sharp absorption peak was obtained using $\mathrm{Al}$ and $\mathrm{Ag}$. In the experiment using Ag, the absorption peak at almost the same angle was obtained. These results can be used to obtain plasmonic band-stop filters in the visible and near-UV region.

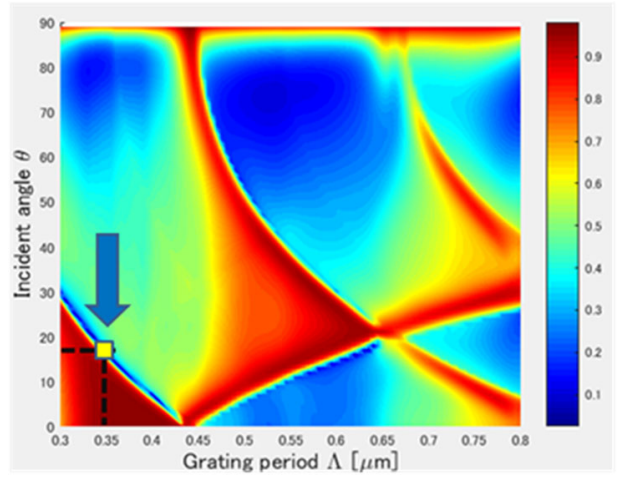

Fig. 3. Reflectance color mapping.

\section{Conclusion}

In this study, we fabricated plasmonic band-stop filters for visible and near-UV light using a WG structure. The results demonstrate that plasmonic band-stop filters in the visible and near-UV region can be realized at $450 \mathrm{~nm}$ and $375 \mathrm{~nm}$ using Ag or Al.

\section{Acknowledgements}

We would like to thank Prof. Takeshi Kato and Dr. Daiki Ohshima for using EBL system. We also thank Prof. Yoshimasa Kawata and Prof. Wataru Inami for using UV laser. This work was conducted at Nagoya University and supported by the Nanotechnology Platform Program of the Ministry of Education, Culture, Sports, Science and Technology (MEXT), Japan, Grant Number JPMXP09F19NU0018. This work was partially supported by JSPS KAKENHI Grant Number 20K05359. We also thank Enago (www.enago.jp) for English language editing.

\section{References}

1. A. Motogaito, Y. Morishita, H. Miyake, K. Hiramatsu, Plasmonics 10, 1657-1662 (2015)

2. A. Motogaito, T. Nakajima, H. Miyake and K. Hiramatsu, Appl. Phys. A 123, 729 (2017)

3. J. Hu, M. Shen, Z. Li, X. Li, G. Liu, X. Wang, C. Kan, Y. Li, Nanotechnology 28, 215205 (2017)

4. Z. Jaksic, M. Maksimovic, M. Sarajlic, D. Tanaskovic, Acta Phys. Pol. A 112, 953-958 (2007)

5. D. B. Mazulquim, K. J. Lee, J. W. Yoon, L. V. Muniz, B.-H. V. Borges, L. G. Neto, R. Magnusson, Opt. Exp. 22, 30843-30851 (2014)

6. C.J. Liang, K.Y. Huang, L.T. Hung, C.Y. Su, Surface \& Coatings Technology 319, 170-181 (2017) 